Cite this: RSC Advances, 2013, 3, 15670

Received 13th March 2013, Accepted 24th June 2013

DOI: $10.1039 / \mathrm{c} 3 \mathrm{ra} 41223 \mathrm{~g}$

www.rsc.org/advances

\section{In situ hard X-ray microscopy of self-assembly in colloidal suspensions $\dagger$}

\author{
Dmytro V. Byelov, ${ }^{\text {*a }}$ Janne-Mieke Meijer, ${ }^{a}$ Irina Snigireva, ${ }^{\mathrm{b}}$ Anatoly Snigirev, ${ }^{\mathrm{b}}$ \\ Laura Rossi, ${ }^{a}$ Esther van den Pol, ${ }^{a}$ Anke Kuijk, ${ }^{c}$ Albert Philipse, ${ }^{a}$ Arnout Imhof, \\ Alfons van Blaaderen, ${ }^{c}$ Gert Jan Vroege ${ }^{a}$ and Andrei V. Petukhov ${ }^{a}$
}

\section{Introduction}

Great efforts in nanoscale science and technology nowadays are focused on the fabrication of functional materials and devices in which the building blocks and their spatial arrangement are engineered down to the nanometre level. Self-assembly of colloids of various shapes governed by complex interactions is an enormous resource for the creation of new functional nanomaterials. For instance, self-assembly allows for constructing novel metamaterials with unprecedented optical, magnetic, electronic, mechanical and rheological properties. ${ }^{1-7}$

Detailed characterization of the long-range periodic order and the structure of local defects in these new materials are inevitable for further progress in applications. Small-angle scattering is a well-established technique to yield ensembleaveraged information on the spatial correlations and longrange order. ${ }^{8-12}$ Direct-space imaging is complementary to scattering because it reveals the local structure of materials and can confirm an interpretation of the scattering data. Common direct-space techniques for colloidal systems study are electron microscopy and (confocal) optical microscopy. ${ }^{13,14}$

\footnotetext{
${ }^{a}$ Van't Hoff Laboratory for Physical and Colloid Chemistry, Debye Institute for Nanomaterials Science, Chemistry Department, Utrecht University, Padualaan 8 , 3584 CH Utrecht, The Netherlands. E-mail: dbyelov@gmail.com

${ }^{b}$ European Synchrotron Radiation Facility, 6 rue Jules Horowitz, F-38043 Grenoble Cedex 9, BP 220, France

${ }^{c}$ Soft Condensed Matter, Debye Institute for Nanomaterials Science, Utrecht University, Princetonplein 1, 3584 CC Utrecht, The Netherlands

$\dagger$ Electronic supplementary information (ESI) available. See DOI: 10.1039/ c3ra41223g
}

These have numerous benefits along with obvious limitations, especially for the in situ studies of (wet) colloidal systems.

Transmission and scanning electron microscopy (TEM/ SEM) provide very high resolution but are essentially ex-situ and limited to either very thin samples (TEM) or surface imaging only (SEM), since the depth of penetration of electrons is very short. Conventional optical microscopy and laser scanning confocal microscopy give information about local structures and dynamics in colloidal systems in situ. ${ }^{15}$ The weak point of optical microscopy is its limited resolution (at best $250-300 \mathrm{~nm}$ ), which strongly reduces the range of applications. A partial solution is provided by several superresolution optical microscopy techniques that go beyond the diffraction limit. ${ }^{16-18}$ However, these optical methods require high transparency of the samples, close matching of particlesolvent refractive indexes and fluorescent labelling. Such requirements are difficult to meet for a wide range of systems like infiltrated photonic crystals or opaque samples.

$\mathrm{X}$-rays is able to fill the gap between electron and optical microscopy. Their shorter wavelength lift restrictions related to diffraction limitations. Their higher penetration power is advantageous in comparison with electron microscopy, especially for in situ studies in wet systems. ${ }^{19-23} \mathrm{X}$-rays can be used for practically all materials and they do not require index matching or fluorescent labelling. An X-ray microscopy setup requires specific optical elements. One of the options is to use Fresnel zone plates as the focusing elements for soft X-rays with energy close to the absorption edge of one of the elements present in the system. The microscopy scheme can be realized in scanning mode - so called scanning transmission X-ray microscopy (STXM) ${ }^{24-26}$ or in full-field mode (TXM). ${ }^{27,28}$ With 
modern Fresnel zone plates one can achieve element-specific imaging with a resolution on the order of about $10 \mathrm{~nm}$ in STXM and in TXM. ${ }^{29}$ Nevertheless, the penetration depth here is usually limited to tenths of micrometres, which is advantageous in comparison to electron. microscopy but still insufficient for bulky, highly adsorbing samples. Resent advances in applications of Fresnel zone plates for the hard X-ray microscopy are given in ref. 30. A "lensless" imaging technique of coherent diffraction imaging (CDI) ${ }^{31,32}$ relies on the phase rather than absorption contrast and can therefore be directly implemented for X-rays with higher photon energy providing a larger penetration depth. However, it requires finite-sized sample objects and the image reconstruction involves complex algorithms with still limited reliability.

In this work we apply full field transmission hard X-ray microscopy ${ }^{33}$ for the in situ imaging of self-assembled colloidal structures in thick (up to 200 microns) wet samples. The setup consists of two sets of compound refractive lenses (CRL) $)^{34,35}$ for beam focusing (condenser) and for projecting a magnified image of the sample on a 2D detector (objective). The setup can be easily switched from microscopy to diffraction mode ${ }^{11,36,37}$ simply by moving the objective CRL out of the beam. We demonstrate that access to both direct and reciprocal space yields complementary structural information for colloidal self-assembled structures.

\section{Experimental}

\subsection{Transmission X-ray microscopy and $\mu \operatorname{radXRD}$}

The X-ray microscopy experiments were performed at the Micro-optics Test Bench installed at the beam line ID06 at the European Synchrotron Radiation Facility (ESRF) in Grenoble. A schematic drawing of the setup is shown in Fig. 1. The experiments were performed at an X-ray energy of $12.2 \mathrm{keV}$ which was selected by a silicon double crystal monochromator with a bandwidth of $\Delta \lambda / \lambda \sim 10^{-4}$. The samples were placed at $57.7 \mathrm{~m}$ from the source and $2 \mathrm{D}$ detectors were placed at 65.5 $\mathrm{m}$. The switching of the setup between diffraction and imaging mode was done by changing the number of refractive lenses in the beam and selecting the appropriate detector. All CRLs and detectors in our setup were installed on motor controlled stages which allowed the switching between modes in a few seconds.

For the diffraction mode the (condenser) CRL consisted of 8 beryllium parabolic lenses with a radius of the parabola apex of $300 \mu \mathrm{m}$ and one lens with a radius of the parabola apex of $1000 \mu \mathrm{m}$. This CRL focused the X-ray beam at a CCD detector (Photonic Science VHR $1 \times 1$ ), with a $9 \times 9 \mu \mathrm{m}^{2}$ pixel size and dimensions of $4008 \times 2671$ pixels. The beam spot size at the sample was $200 \times 200 \mu \mathrm{m}^{2}$.

For the imaging mode additional CRLs were moved into the beam. One set of CRLs for focusing the beam at the sample was inserted at 38.7 meters from the source (not shown in Fig. 1) providing a beam spot size of $50 \times 50 \mu \mathrm{m}^{2}$ at the sample. Another CRL consisting of 35 beryllium lenses, with a radius of the parabola apex of $50 \mu \mathrm{m}$ was inserted at $30 \mathrm{~cm}$ after the sample as the objective. A high resolution CCD detector ESRF FReloN 2000 with $0.56 \times 0.56 \mu \mathrm{m}^{2}$ pixel size and dimensions of $2048 \times 2048$ pixels was employed for the imaging mode. This configuration provides a magnification about 22 as was estimated from the geometry of the setup and confirmed by measurements on a tungsten calibration grid. The spatial resolution of our microscopy setup we estimated by resolving structure of the Tantalum Siemens star with a pitch of $100 \mathrm{~nm}$ and thickness of $0.5 \mu \mathrm{m}$.

\subsection{Particle synthesis}

2.2.1 Silica rods (Samples R1 and R2). Silica rod-like particles (Fig. 2A) were synthesized following a method published earlier. ${ }^{38}$ To a solution of polyvinylpyrrolidone (PVP) in pentanol a mixture of ethanol, water, sodium citrate was added, resulting in an emulsion. From the emulsion droplet, silica rod-like particles were grown upon the addition of tetra-ethyl orthosilicate (TEOS) resulting in a bullet-like particle shape (Fig. 2A). The particle length $L$ and diameter $D$ were characterized by TEM and the data are shown in Table 1 . For the experiments, particles were concentrated to volume

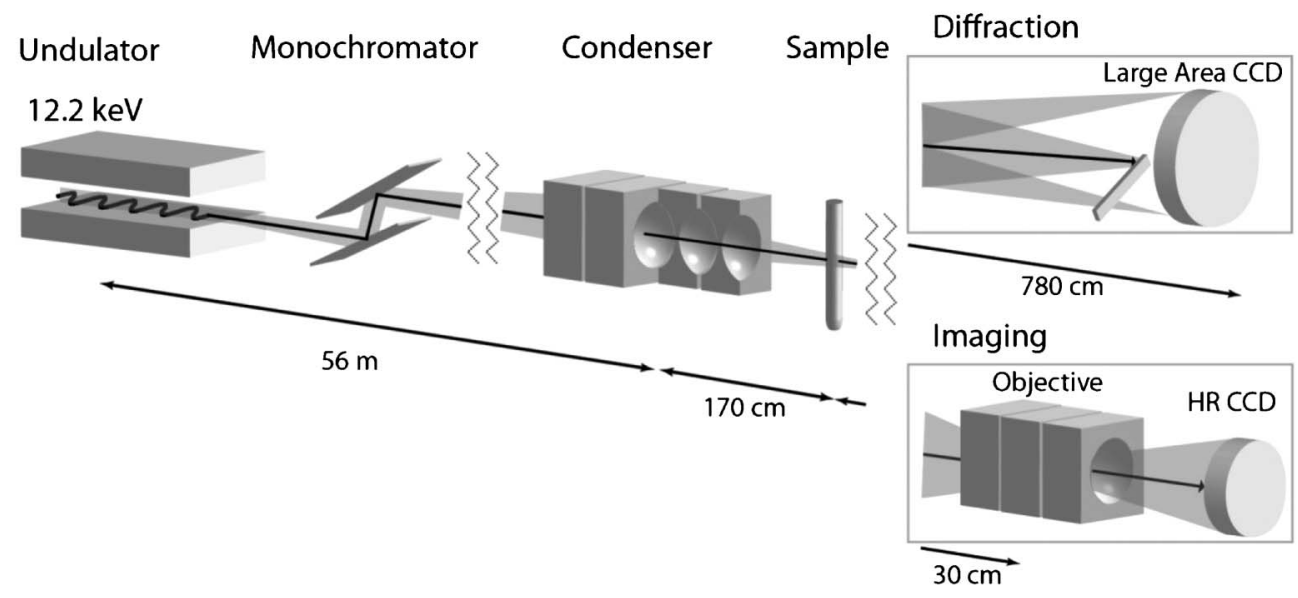

Fig. 1 Schematic representation of the $\mu$ radXRD and theTXM setups. The extended version is presented in the ESI.† 


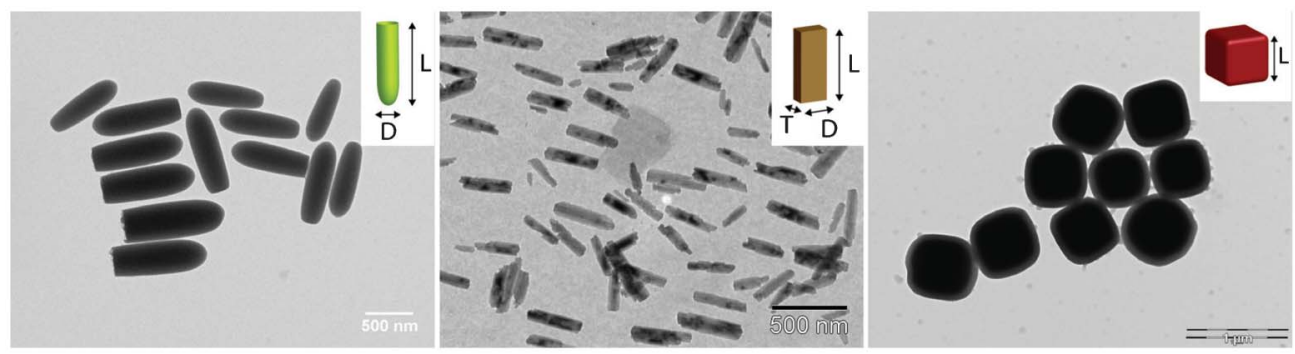

Fig. 2 TEM images and schematic representation of the studied colloidal systems: A) silica rods, B) goethite board-like particles and C) hematite cubes.

fractions of $\sim 10-30$ vol\% in water and placed in $100 \mathrm{~mm}$ long flat glass capillaries (Vitrocom RT5015) with internal dimensions of $0.05 \mathrm{~mm}$ by $1.0 \mathrm{~mm}$. The capillaries were flame sealed. Studies were performed in the bottom part of the capillaries, where close packing of particles was achieved due to sedimentation.

2.2.2 Goethite dispersion (Sample B1). Goethite $(\alpha-\mathrm{FeOOH})$ particles were obtained by hydrolysis of iron nitrate at high $\mathrm{pH}$, details on the synthesis can be found in. ${ }^{39}$ In short, $1 \mathrm{M}$ $\mathrm{NaOH}$ was added dropwise, under stirring, to a $0.1 \mathrm{M} \mathrm{Fe}\left(\mathrm{NO}_{3}\right)_{3}$ solution until a $\mathrm{pH}$ of 11-12 was reached. The precipitate was aged for 9 days at room temperature. Particles with a boardlike shape were obtained and concentrated in water to a volume fraction of $12 \%$. In Fig. 2B a TEM image and schematic picture of the particles are shown. The length $L$, width $D$ and thickness $T$ together with standard deviations for the length $\sigma_{\mathrm{L}}$ and for the width $\sigma_{\mathrm{D}}$ were determined from TEM and summarized in Table 1. The samples were placed in $100 \mathrm{~mm}$ long flat borosilicate capillaries with internal dimensions of $0.2 \mathrm{~mm}$ by $4.0 \mathrm{~mm}$. The capillaries were flame sealed or closed with two-component epoxy glue (Bison Kombi rapide) and kept in a vertical position to allow the establishment of a sedimentation equilibrium profile.

2.2.3 Silica coated hematite cubes (Sample C1). Colloidal hematite $\left(\alpha-\mathrm{Fe}_{2} \mathrm{O}_{3}\right)$ cubes were prepared from a condensed ferric hydroxide gel and coated with an amorphous silica shell as described in detail elsewhere. ${ }^{2,3,40}$ In brief, hematite cubes with a typical edge length of $519 \mathrm{~nm}$ were prepared from a 2.0 $\mathrm{M} \mathrm{FeCl}_{3} \cdot 6 \mathrm{H}_{2} \mathrm{O}$ solution which was slowly mixed with a $6.2 \mathrm{M}$ $\mathrm{NaOH}$ solution to a $\mathrm{pH}$ of 14 and aged for 8 days at $100{ }^{\circ} \mathrm{C}$. The cubes were coated with a $67 \mathrm{~nm}$ silica layer according to the Stöber method resulting in a particle edge length of $652 \mathrm{~nm}$ (Table 1). ${ }^{41}$ The silica layer can be seen on the TEM image as the lighter grey edge around the cubes in Fig. 2C. The particles were transferred to ethanol and concentrated to volume fractions of $\sim 5 \mathrm{v} \%$. For the X-ray measurements flat

Table 1 Overview of particle dimensions of the different colloidal systems

\begin{tabular}{llllllll}
\hline System & $L(\mathrm{~nm})$ & $\begin{array}{l}\sigma_{\mathrm{L}} \\
(\%)\end{array}$ & $\begin{array}{l}D \\
(\mathrm{~nm})\end{array}$ & $\begin{array}{l}\sigma_{\mathrm{D}} \\
(\%)\end{array}$ & $\begin{array}{l}T \\
(\mathrm{~nm})\end{array}$ & $L / D$ & $\begin{array}{l}\text { vol } \\
(\%)\end{array}$ \\
\hline Silica Rods - R1 & 940 & 6 & 300 & 12 & - & 3.1 & 23 \\
Silica Rods - R2 & 1950 & 7 & 250 & 11 & - & 7.7 & 12.8 \\
Goethite Boards - B1 & 282 & 35 & 68 & 32 & $\sim 25$ & 4.1 & 12.4 \\
Hematite Cubes - C1 & 652 & 7 & - & - & - & - &
\end{tabular}

borosilicate rectangular capillaries (internal cross section of $0.2 \mathrm{~mm}$ by $4.0 \mathrm{~mm}$ ) were used, the same as for Goethite dispersions. Crystal formation in the samples was observed in the sediment within 1 day of sedimentation, indicated by distinct Bragg reflections. For sedimentation in a magnetic field, the samples were redispersed by repeated manual tumbling and 5-10 min ultrasonic treatment. Then samples were placed in a magnetic field of $24 \mathrm{mT}$ for at least $12 \mathrm{~h}$ until the presence of crystalline sediment was observed.

\section{Results and discussion}

Colloidal dispersions in capillaries were subjected to a gravity field in order to establish a sedimentation equilibrium profile. For goethite and hematite particles an external magnetic field was also applied to orient the self-assembled structures. Different liquid crystal phases were found over the length of capillaries. The averaged morphology of the structures formed was first examined by $\mu \mathrm{radXRD}$ to locate regions of interest and to estimate characteristic periodicities. The regions were then investigated with TXM to get insight into the local realspace morphology of the samples and to clarify the structure.

\subsection{Silica rods}

In Fig. 3A a diffraction pattern from the bottom part of the silica rods sample with $L=940 \mathrm{~nm}$ and $D=300 \mathrm{~nm}$ is shown (sample R1). The pattern is characteristic for smectic ordering of the particles. The vertical set of sharp peaks in the low $q$-range; at $q_{1}=0.0061 \mathrm{~nm}^{-1}, q_{2}=0.0122 \mathrm{~nm}^{-1}$ and $q_{3}=0.0178$ $\mathrm{nm}^{-1}$, originate from the smectic periodicity. Their $q$-values are related as $1: 2: 3$. The smectic period was found to be $2 \pi /$ $q_{1}=1030 \mathrm{~nm}$, which is comparable to the averaged particle length $L$ of $940 \mathrm{~nm}$. The vertical peaks are much broader in the azimuthal direction than in the radial direction, indicating a strong variation in the orientation of the smectic layers while keeping the smectic period fixed. In the horizontal direction of the diffraction pattern, a set of broader peaks is observed. Peaks at $q_{1}=0.0177 \mathrm{~nm}^{-1}$ and $q_{2}=0.0305 \mathrm{~nm}^{-1}$ originate from the ordering of rods within the smectic layers. These $q$-values are related as $1: \sqrt{3}$, which points to an only shortrange hexagonal arrangement of the rods within the smectic layers.

The TXM image of the same region (Fig. 3B) supports the interpretation made on basis of the diffraction data. The 


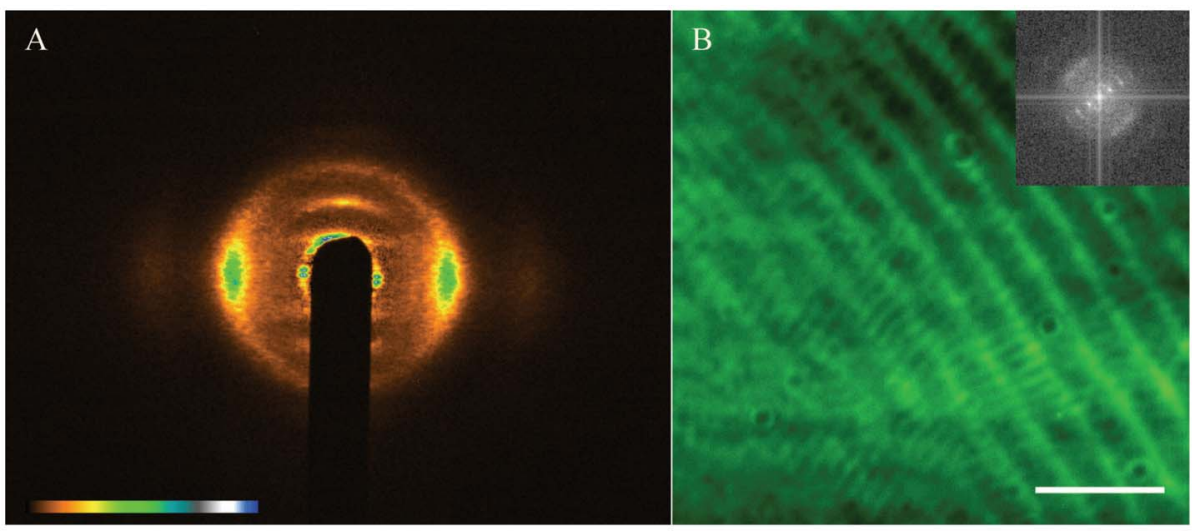

Fig. 3 A) $\mu$ radXRD pattern and B) TXM image of a smectic phase formed in the sediment of silica rods R1 with the aspect ratio of 3.1. In panel A the scattering intensity is shown on a logarithmic scale within one decade using the false colour table shown. The corresponding /(q) profiles are presented in the ESI. $\uparrow$ The image in B exploits monochrome linear intensity scale. White scale bar in B) indicates $3 \mu \mathrm{m}$. The inset in B) presents a Fourier transform of the image; vertical and horizontal stripes are the artefacts of the transformation.

interlayer smectic periodicity and the strong in-plane positional correlations between the particles are clearly visible. One can also observe variations in the orientation of the smectic layers. The Fourier transform of the TXM image is shown in the inset and corresponds well to the diffraction image. It has to be noted that in the used 2D discrete Fourier transform the signal function was assumed to be periodic in both directions which caused artifacts like bright lines along the spectrum's axis.

In Fig. 4A a $\mu$ radXRD pattern of a smectic phase formed in a dispersion of silica rods with length $L=1950 \mathrm{~nm}$ and diameter $D=250 \mathrm{~nm}$ is shown (sample R2). Peaks in the low $q$-region at $q_{2}=0.0056 \mathrm{~nm}^{-1}, q_{3}=0.0083 \mathrm{~nm}^{-1}, q_{4}=0.0112 \mathrm{~nm}^{-1}$ and $q_{5}=$ $0.0140 \mathrm{~nm}^{-1}$ are related as $2: 3: 4: 5$ and originate from the smectic ordering in the system with a periodicity of $2.24 \mu \mathrm{m}$. The first-order peaks are hidden behind the beamstop. The broad peak at $q_{1}=0.0168 \mathrm{~nm}^{-1}$ originates from liquid-like ordering of the rods within smectic layers. The diffraction peaks originating from correlations in length are not exactly orthogonal to the peaks originating from correlations between neighboring particles within the smectic layers. This implies that this system possessed a smectic-C phase, ${ }^{42}$ in which the rods within the layers were inclined at an angle of $\sim 12^{\circ}$ with respect to the layer's normal.

In Fig. 4B the TXM image is shown, it illustrates the morphology of the system in real space and includes the inclination of the rods to the layer normal. The Fourier transform of the image in the inset of Fig. $4 \mathrm{~B}$ is identical to the diffraction pattern and demonstrates the great correspondence between the local real-space structures observed with TXM and the much more averaged long range morphology probed by $\mu$ radXRD. Further real space analysis of these samples with quantitative confocal microscopy on a single rod level is also in accordance with the X-ray results presented here and are given in ref. 43.

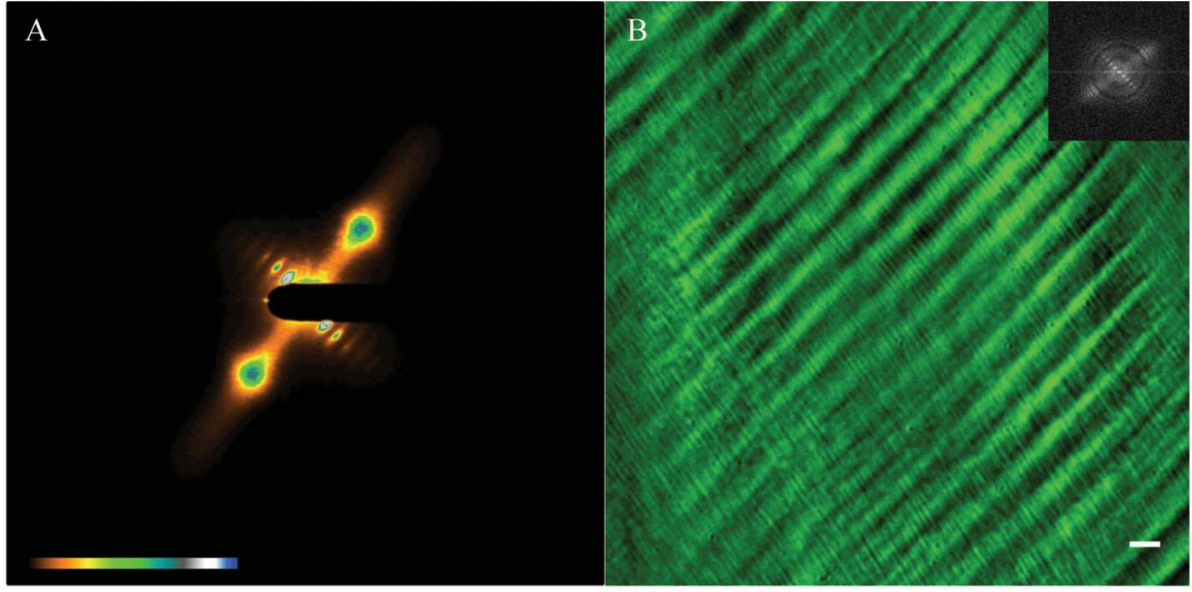

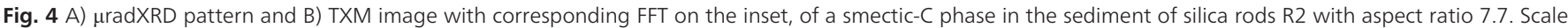
bar in B) indicates $3 \mu \mathrm{m}$. See caption of Fig. 1 for colour encoding. 


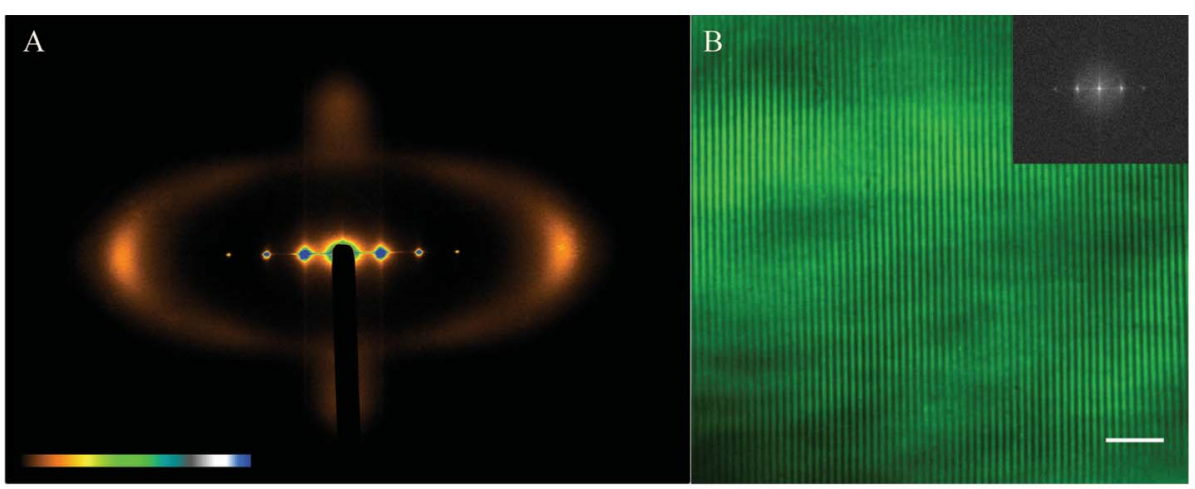

Fig. 5 Highly ordered smectic phase of goethite rods with aspect ratio (L/D) 4.1 aligned in an applied magnetic field of 200 mT: A) the $\mu$ radXRD pattern (the

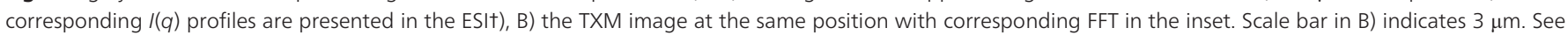
caption of Fig. 1 for colour encoding.

\subsection{Goethite boards}

A system which is very appealing for study with a combined $\mathrm{TXM} / \mu \mathrm{radXRD}$ method is a dispersion of colloidal goethite board-like particles. This system is opaque and hard to index match. Moreover, it has dimensions significantly smaller than the previous anisotropic model system, making it unsuitable for studies with conventional microscopy methods and therefore commonly investigated with X-ray scattering techniques. It was found that these board-like particles can form various liquid crystal phases, such as nematic, smectic and columnar, depending on the particle aspect ratio, polydispersity and concentration in solution. ${ }^{44,45}$ The particles possess a permanent magnetic moment along their long axis $L$, together with an induced magnetic moment along the shortest particle axis $T$. Thus, when a weak external magnetic field is applied the particles orient parallel to the magnetic field and upon increasing the field the particles reorient perpendicular to the field. ${ }^{46}$ It has also been found that phase transitions occur at elevated fields. ${ }^{47-49}$

Here we study the behaviour of sample B1 subjected to a magnetic field. When a small magnetic field of about $200 \mathrm{mT}$ is applied, the particles orient along the field and the smectic phase becomes highly oriented. With $\mu$ radXRD average qualitative and quantitative information on the structure within the sample was obtained. In Fig. 5A the diffraction pattern of the sample in a horizontally oriented field of $200 \mathrm{mT}$ is shown. Three sharp peaks at small angles $\left(q_{1}=0.0165 \mathrm{~nm}^{-1}\right.$, $q_{2}=0.0331 \mathrm{~nm}^{-1}$ and $q_{3}=0.0497 \mathrm{~nm}^{-1}$ ) in the horizontal direction are related to each other as $q_{1}: \mathrm{q}_{22}: q_{3}=1: 2: 3$ and originate from the smectic layering formed in the system. The horizontal orientation of the peaks indicates that the smectic layer's normal is oriented along the field direction. In the vertical direction a broad peak at $q=0.0800 \mathrm{~nm}^{-1}$ is present due to liquid-like ordering of particles within the smectic layers. A broad horizontal peak at $q=0.0954 \mathrm{~nm}^{-1}$ indicates that the magnetic field was strong enough to also reorient some particles perpendicular to the field direction. ${ }^{48}$

The corresponding TXM picture is shown in Fig. 5B. The period between the vertical lines is about $380 \mathrm{~nm}$, which is in agreement with the average smectic spacing obtained from diffraction $2 \pi / q_{1}=381 \mathrm{~nm}$. In the inset of Fig. 5B the Fourier transform of the TXM image is shown which corresponds qualitatively and quantitatively well to the diffraction pattern. Unfortunately, individual particles within the layers cannot be resolved with TXM, because the inter-particle spacing was on the order of $70 \mathrm{~nm}$, which is close to the resolution limit of our setup. Moreover, since thick samples were studied, the projection of many particles with short range ordering smears out images of the individual particles.

When the magnetic field was increased above $280 \mathrm{mT}$, the particles reoriented from parallel to perpendicular to the field. This is clearly seen in Fig. 6B, where a TXM pattern of the resulting structure is shown. In the diffraction pattern (Fig. 6A) only one sharp horizontal peak at low $q=0.0165 \mathrm{~nm}^{-1}$ is present without any sign of the higher order peaks and a very intense broad peak at the same $q$ value appears in the vertical direction. All this proves the reorientation of the smectic layers. The layers now possessed undulations as the long-range order was lost and the particles actually seem to have partly rearranged into a columnar phase. Four peaks at $q=0.064$ $\mathrm{nm}^{-1}$ and two peaks at $q=0.093 \mathrm{~nm}^{-1}$ can be observed.

In Fig. 6B the TXM image is shown and the morphology of the sample is visualized in real space. Here, the layers are rotated by 90 degrees and form undulating ribbon-like structures. When compared to Fig. $4 \mathrm{~B}$ it is clear that the perfect ordering of the smectic layers has disappeared. The Fourier transform of the image (Fig. 6B inset) matches the low $\mathrm{q}$ region of the diffraction pattern.

Although the $\operatorname{radXRD}$ shows the presence of multiple domains and a columnar phase in the sample, here the TXM gives more insight into the local morphology of the system. This is a good example why a combination of the two techniques is important in obtaining the 'complete' picture.

\subsection{Hematite cubes}

The suitability of the combined techniques for in situ study became most evident in the study of sedimented silica-coated hematite cubes in a magnetic field. These iron oxide cubes possess a magnetic moment and align in the horizontal magnetic field. The particles have rounded corners and can be described by a so-called superball model $\left|x_{1}\right|^{m}+\left|x_{2}\right|^{m}+\left|x_{3}\right|^{m}$ 


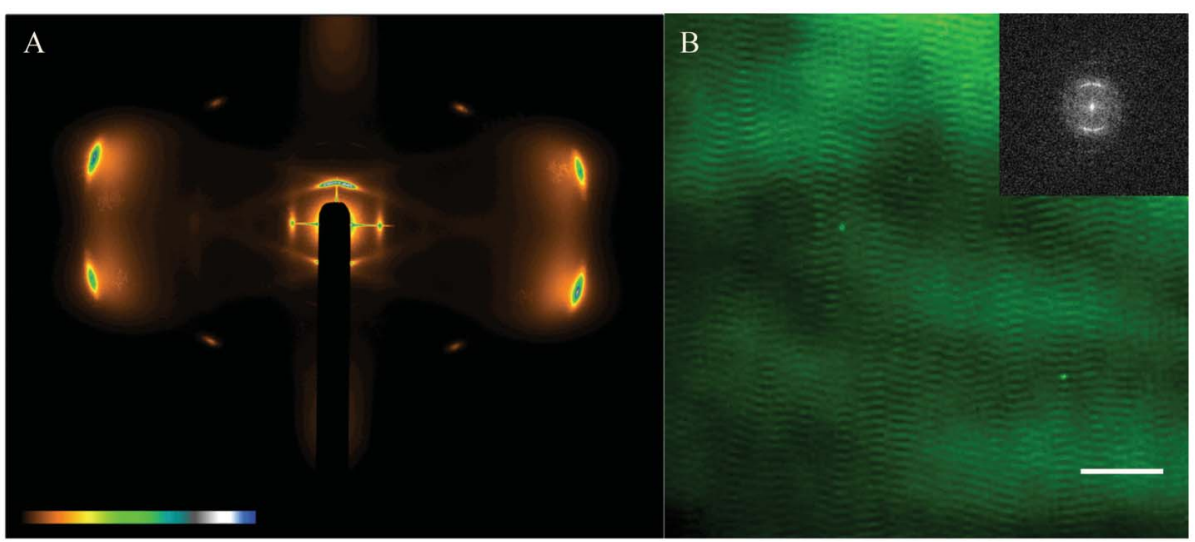

Fig. 6 Reoriented smectic phase in the increased magnetic field $280 \mathrm{mT}$ : A) the $\mu$ radXRD pattern; B) TXM image with corresponding FFT in the inset. Scale bar in B) indicates $3 \mu \mathrm{m}$. See caption of Fig. 1 for colour encoding.

$\leqslant 1$. From an analysis of TEM images of individual particles it was found that $m=3.2$. As was predicted in simulations ${ }^{50}$ the optimal packing of such particles is not a simple cubic structure but a distorted face centred cubic (FCC) lattice- $\mathrm{C}_{1}$ phase.

With $\mu$ radXRD measurements we have found three different domains represented by isotropic, square and hexagonal patterns. In Fig. 7A a $\mu$ radXRD pattern which we assigned to the simple cubic phase is shown. Sharp peaks from the cubic ordering (see model structure in the inset) with a typical spacing of $580 \mathrm{~nm}$ are present. In Fig. 7B the interface between an isotropic phase and a cubic phase is visualized by TXM (FFT of the image is shown in the inset). Good contrast of the image in the cubic phase region points towards a high degree of ordering of the sample along the beam. The ordered cubic domains were present along the whole width $(4 \mathrm{~mm})$ of the capillary at fixed height. In the vertical direction it spans approximately the whole TXM image which is about $50 \mu \mathrm{m}$. The Fourier transform of the TXM image (inset Fig. 7B) corresponds to the $\mu \mathrm{radXRD}$ pattern (Fig. 7A).
A hexagonal-type arrangement appeared below the cubic phase in the capillary. In Fig. 8 the $\mu$ radXRD pattern (A) with a simulated structure (inset Fig. 8A) and TXM image (Fig. 8B) with its Fourier transform (inset Fig. 8B) are shown. The simulated $\mathrm{C}_{1}$ structure provides a similar distribution of peaks in $q$-space corresponding to the measured $\mu \mathrm{radXRD}$ pattern, which confirms the formation of this phase.

\section{Conclusions}

We have shown that full field transmission X-ray microscopy is a very suitable technique for the in situ study of self-assembled colloidal systems. Using both $\mu \operatorname{radXRD}$ and TXM we have studied self-assembled structures formed in dispersions. The local morphology of a smectic phase formed in dispersions of micron-sized silica rods could be visualized in real space by the TXM technique. This includes variation in the orientation of the smectic layers while keeping the smectic period fixed

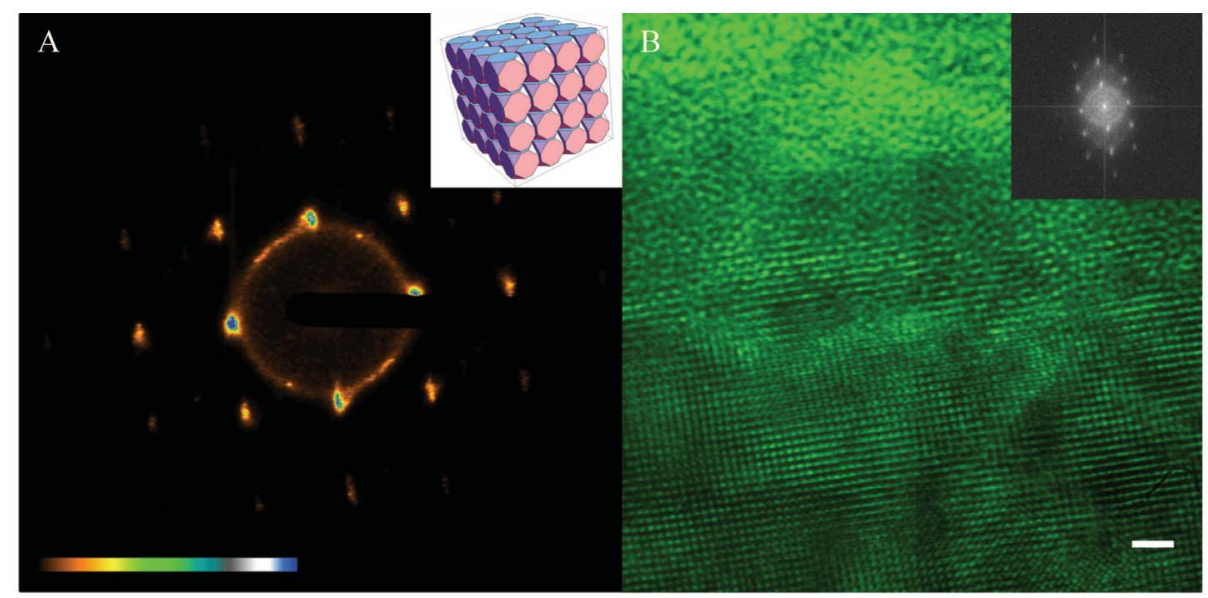

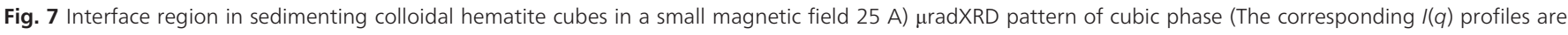

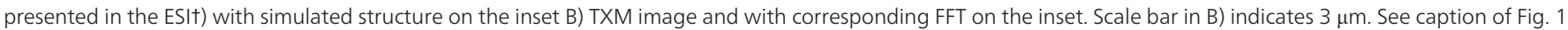
for colour encoding. 


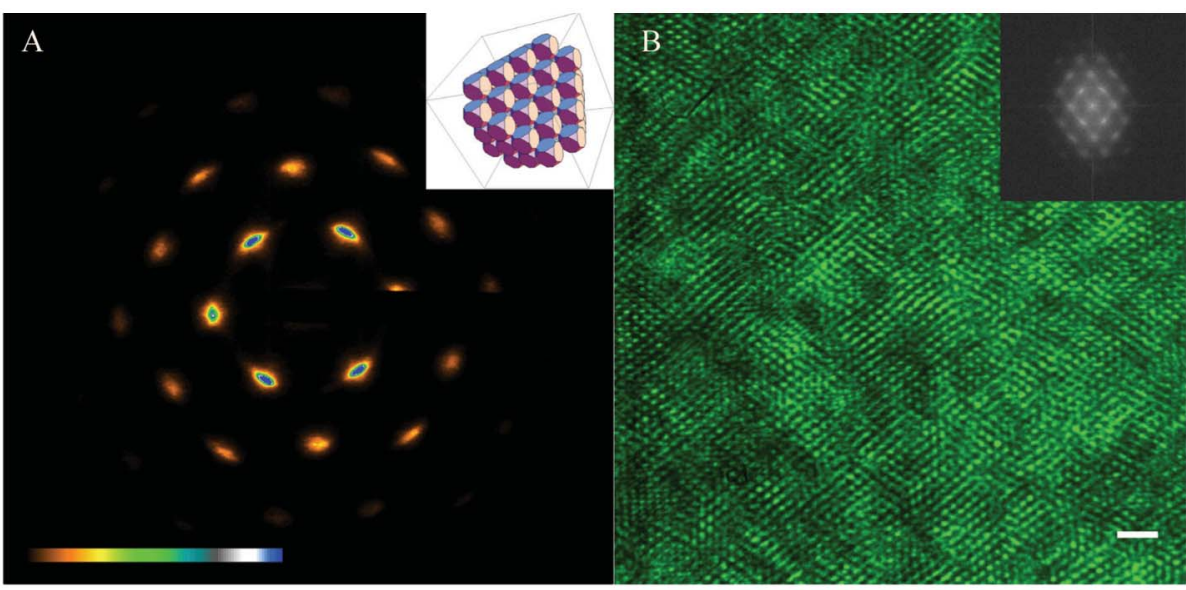

Fig. 8 A) $\mu \mathrm{radXRD}$ pattern with simulated structure and B) TXM image with Fourier transform of a distorted FCC phase formed in the sediment of colloidal cubes in a magnetic field of $25 \mathrm{mT}$. Scale bar in B) indicates $3 \mu \mathrm{m}$. See caption of Fig. 1 for colour encoding.

and a 12 degree inclination of the particles to the smectic normal within the smectic layers. For the significantly smaller goethite board-like particles a better understanding of the reorientation behavior of the smectic phase, in a high magnetic field, was obtained, clearly demonstrating the complementary information obtained by both techniques: TXM reveals the zig-zag pattern of the reorienting smectic, while only $\mu \mathrm{radXRD}$ reveals the simultaneous formation of a columnar phase. The most prominent example is the visualization of the cubic phase at the order-disorder interface formed by sedimented hematite cubes and the appearance of a $\mathrm{C}_{1}$ phase below it. It is important to highlight that the study of the interface in real space was done without any special sample modifications.

The results clearly illustrate that the hard X-ray TXM technique has great potential for in situ studies of colloidal self-assembly. The combination of the direct-space imaging and microradian diffraction is able to provide a wealth of information on their local structure (imaging) and the longrange periodic order (diffraction). Future possible applications of the technique include time-resolved studies of the crystallization dynamics, response of the colloidal assembly to external stimuli such as mechanical strain, temperature jump or temperature gradient as well as external fields.

\section{Acknowledgements}

The European Synchrotron Radiation Facility (ESRF) in Grenoble is acknowledged for the provided beam-time. C. Detlefs and T. Roth from ID06 are gratefully thanked for the support during experiments.

\section{References}

1 V. F. Puntes, K. M. Krishnan and A. P. Alivisatos, Science, 2001, 291, 2115-2117.
2 L. Rossi, S. Sacanna, W. T. M. Irvine, P. M. Chaikin, D. J. Pine and A. P. Philipse, Soft Matter, 2011, 7, 4139-4142.

3 L. Rossi, Utrecht University, 2012.

4 P. Davidson and J. C. P. Gabriel, Curr. Opin. Colloid Interface Sci., 2005, 9, 377-383.

5 F. Romano and F. Sciortino, Nat. Mater., 2011, 10, 171-173.

6 E. van den Pol, A. V. Petukhov, D. M. Thies-Weesie, D. V. Byelov and G. J. Vroege, Phys. Rev. Lett., 2009, 103, 258301-258304.

7 A. P. Hynninen, J. H. Thijssen, E. C. Vermolen, M. Dijkstra and A. van Blaaderen, Nat. Mater., 2007, 6, 202-205.

8 W. L. Vos, M. Megens, C. M. van Kats and P. Bosecke, Langmuir, 1997, 13, 6004-6008.

9 A. B. D. Brown, S. M. Clarke and A. R. Rennie, Langmuir, 1998, 14, 3129-3132.

10 F. M. van der Kooij, K. Kassapidou and H. N. W. Lekkerkerker, Nature, 2000, 406, 868-871.

11 A. V. Petukhov, J. H. J. Thijssen, D. C. t. Hart, A. Imhof, A. van Blaaderen, I. P. Dolbnya, A. Snigirev, A. Moussaid and I. Snigireva, J. Appl. Crystallogr., 2006, 39, 137-144.

12 N. A. Grigoryeva, A. A. Mistonov, K. S. Napolskii, N. A. Sapoletova, A. A. Eliseev, W. Bouwman, D. V. Byelov, A. V. Petukhov, D. Y. Chernyshov, H. Eckerlebe, A. V. Vasilieva and S. V. Grigoriev, Phys. Rev. B: Condens. Matter Mater. Phys., 2011, 84, 064405-064418.

13 H. Friedrich, C. J. Gommes, K. Overgaag, J. D. Meeldijk, W. H. Evers, B. de Nijs, M. P. Boneschanscher, P. E. de Jongh, A. J. Verkleij, K. P. de Jong, A. van Blaaderen and D. Vanmaekelbergh, Nano Lett., 2009, 9, 2719-2724.

14 A. van Blaaderen and P. Wiltzius, Science, 1995, 270, 1177-1179.

15 V. Prasad, D. Semwogerere and E. R. Weeks, J. Phys.: Condens. Matter, 2007, 19, 113102-113127.

16 S. W. Hell, Science, 2007, 316, 1153-1158.

17 S. W. Hell, Nat. Methods, 2009, 6, 24-32.

18 Z. B. Wang, W. Guo, L. Li, B. Luk'yanchuk, A. Khan, Z. Liu, Z. C. Chen and M. H. Hong, Nat. Commun., 2011, 2, 218-224.

19 K. Kaznacheev and T. Hegmann, Phys. Chem. Chem. Phys., 2007, 9, 1705-1712. 
20 J. Thieme, I. McNulty, S. Vogt and D. Paterson, Environ. Sci. Technol., 2007, 41, 6885-6889.

21 S. Brisard, R. S. Chae, I. Bihannic, L. Michot, P. Guttmann, J. Thieme, G. Schneider, P. J. M. Monteiro and P. Levitz, Am. Mineral., 2012, 97, 480-483.

22 J. M. Sieliechi, B. S. Lartiges, G. J. Kayem, S. Hupont, C. Frochot, J. Thieme, J. Ghanbaja, J. B. D. de la Caillerie, O. Barres, R. Kamga, P. Levitz and L. J. Michot, Water Res., 2008, 42, 2111-2123.

23 L. J. Michot, I. Bihannic, F. Thomas, B. S. Lartiges, Y. Waldvogel, C. Caillet, J. Thieme, S. S. Funari and P. Levitz, Langmuir, 2013, 29, 3500-3510.

24 H. Ade, A. P. Smith, H. Zhang, G. R. Zhuang, J. Kirz, E. Rightor and A. Hitchcock, J. Electron Spectrosc. Relat. Phenom., 1997, 84, 53-71.

25 T. Warwick, H. Ade, A. P. Hitchcock, H. Padmore, E. G. Rightor and B. P. Tonner, J. Electron Spectrosc. Relat. Phenom., 1997, 84, 85-98.

26 T. Warwick, K. Franck, J. B. Kortright, G. Meigs, M. Moronne, S. Myneni, E. Rotenberg, S. Seal, W. F. Steele, H. Ade, A. Garcia, S. Cerasari, J. Delinger, S. Hayakawa, A. P. Hitchcock, T. Tyliszczak, J. Kikuma, E. G. Rightor, H. J. Shin and B. P. Tonner, Rev. Sci. Instrum., 1998, 69, 2964-2973.

27 D. T. Attwood, Soft X-Rays and Extreme Ultraviolet Radiation: Principles and Applications, Cambridge University Press, Cambridge, U.K., 2000.

28 M. Howells, C. Jacobsen, T. Warwick and A. Van den Bos, in Science of Microscopy, ed. P. W. Hawkes and J. C. H. Spence, Springer, New York, 2007, pp. 835-926.

29 W. Chao, P. Fischer, T. Tyliszczak, S. Rekawa, E. Anderson and P. Naulleau, Opt. Express, 2012, 20, 9777-9783.

30 S.-R. Wu, Y. Hwu and G. Margaritondo, Materials, 2012, 5, 1752-1773.

31 I. K. Robinson, I. A. Vartanyants, G. J. Williams, M A. Pfeifer and J. A. Pitney, Phys. Rev. Lett., 2001, 87, 195505-195504.

32 I. A. Vartanyants, I. K. Robinson, J. D. Onken, M. A. Pfeifer, G. J. Williams, F. Pfeiffer, H. Metzger, Z. Zhong and G. Bauer, Phys. Rev. B: Condens. Matter Mater. Phys., 2005, 71, 245302-245311.

33 A. Bosak, I. Snigireva, K. S. Napolskii and A. Snigirev, Adv. Mater., 2010, 22, 3256-3259.
34 A. Snigirev, V. Kohn, I. Snigireva and B. Lengeler, Nature, 1996, 384, 49-51.

35 B. Lengeler, C. G. Schroer, M. Richwin, J. Tummler, M. Drakopoulos, A. Snigirev and I. Snigireva, Appl. Phys. Lett., 1999, 74, 3924-3926.

36 M. Drakopoulos, A. Snigirev, I. Snigireva and J. Schilling, Appl. Phys. Lett., 2005, 86, 014102-014105.

37 D. V. Byelov, M. C. D. Mourad, I. Snigireva, A. Snigirev, A. V. Petukhov and H. N. W. Lekkerkerker, Langmuir, 2010, 26, 6898-6901.

38 A. Kuijk, A. van Blaaderen and A. Imhof, J. Am. Chem. Soc., 2011, 133, 2346-2349.

39 D. M. E. Thies-Weesie, J. P. de Hoog, M. H. H. Mendiola, A. V. Petukhov and G. J. Vroege, Chem. Mater., 2007, 19, 5538-5546.

40 T. Sugimoto, M. M. Khan, A. Muramatsu and H. Itoh, Colloids Surf., A, 1993, 79, 233-247.

41 C. Graf, D. L. J. Vossen, A. Imhof and A. van Blaaderen, Langmuir, 2003, 19, 6693-6700.

42 P. G. de Gennes and J. Prost, The Physics of Liquid Crystals, Second Edition edn, Clarendon Press, 1993.

43 A. Kuijk, D. V. Byelov, A. V. Petukhov, A. van Blaaderen and A. Imhof, Faraday Discuss., 2012, 159, 181-199.

44 B. J. Lemaire, P. Davidson, J. Ferre, J. P. Jamet, D. Petermann, P. Panine, I. Dozov, D. Stoenescu and J. P. Jovilet, Faraday Discuss., 2005, 128, 271-283.

45 G. J. Vroege, D. M. E. Thies-Weesie, A. V. Petukhov, B. J. Lemaire and P. Davidson, Adv. Mater., 2006, 18, 2565-2568.

46 B. J. Lemaire, P. Davidson, J. Ferre, J. P. Jamet, P. Panine, I. Dozov and J. P. Jolivet, Phys. Rev. Lett., 2002, 88, 125507-125511.

47 B. J. Lemaire, P. Davidson, P. Panine and J. P. Jolivet, Phys. Rev. Lett., 2004, 93, 267801-267804.

48 E. van den Pol, A. V. Petukhov, D. V. Byelov, D. M. E. ThiesWeesie, A. Snigirev, I. Snigireva and G. J. Vroege, Soft Matter, 2010, 6, 4895-4899.

49 E. van den Pol, A. Lupascu, P. Davidson and G. J. Vroege, J. Chem. Phys., 2010, 133, 164504-164511.

50 Y. Jiao, F. H. Stillinger and S. Torquato, Phys. Rev. E: Stat., Nonlinear, Soft Matter Phys., 2009, 79, 041309-041321. 